\title{
MARQUEURS DU TRACTUS GÉNITAL ET ACTIVITÉS GLYCOSIDASIQUES SÉMINALES
}

\author{
Jacques Gonzalès*, Saïd Ben Ayed**, Bernard Maguy** et François Percheron** \\ * Unité de Biologie de la Reproduction - Laboratoire d'Histologie Embryologie \\ ** Laboratoire Central de Biochimie - Hôpital de la Pitié, 83, Boulevard de l'Hôpital, 75651 PARIS, Cedex 13.
}

\section{MARKERS OF THE GENITAL TRACT} AND SEMINAL GLYCOSIDASES ACTIVITIES. Seminal liquefaction corresponds to a proteolysis of glycoproteins and could also result in a lysis of linked glycans involving glycosidases. It is well known that $\alpha-1-4$ glucosidase is a real epipidymal marker. The aim of this study is to point out new markers among the glycosidases and especially $\beta-D$ mannosidase, $\alpha$-L-fucosidase and $N$-acetyl- $\beta$ D-glucosaminidase.

There is no correlation between the three enzymatic activities and the sperm count. Moreover in case of azoospermia enzymatic activities were always present. The variations observed are both quantitative ( $\beta$-MAN activity is significantly decreased) and qualitative (modifications of the relative proportions of the different enzymatic forms) according to the etiology of azoospermia.

Key-words : Human seminal plasma Glycosidases - $\alpha$-L-fucosidase - $\beta$-Dmannosidase - $N$-acetyl- $\beta$-D-glucosaminidase Azoospermia.

ANDROLOGIE, 1991, 1:13-14

\section{INTRODUCTION}

La liquéfaction du sperme correspond à une protéolyse touchant notamment des glycoprotéines au niveau de leur "core" peptidique (figure 1)(1).Il se pourrait également que la liquéfaction s'accompagne d'une lyse au niveau des branchements glycaniques impliquant une action d'un autre type d'enzymes, les glycosidases.

La présence des glycosidases dans le plasma séminal a été depuis longtemps démontrée (2). Depuis une dizaine d'années, l' $\alpha 1-4$ glucosidase a fait l'objet de diverses publications (3) dans deux buts essentiels :

- mettre en évidence l'origine sécrétoire de cette enzyme qui pourrait être alors un marqueur supplémentaire du tractus génital, particulièrement dans le cas d'azoospermie excrétoire.

- trouver une corrélation entre l'activité enzymatique et la qualité du spermogramme, en particulier la mobilité des spermatozoïdes.

Au terme de ces travaux, il semble que l' $\alpha-1-4$ glucosidase constitue un bon marqueur épididymaire (4). Il existe en outre une corrélation entre cette activité enzymatique et le pourcentage de spermatozoïdes mobiles (5). L' $\alpha-1-4$ glucosidase constituerait finalement un bon marqueur de la fonction de l'épididyme dont la finalité est pour le spermatozoïde en maturation l'acquisition de la mobilité (6).

Notre étude a pour but de rechercher d'autres marqueurs parmi les glycosidases et plus particulièrement la $\beta$-D-mannosidase ( $\beta M A N)$ (EC 3.2.1.25), l' $\alpha$-L-fucosidase ( $\alpha$-FUC)(EC 3.2.1.51) et la $\mathrm{N}$-acétyl- $\beta$-D-glucosaminidase (NAG) (EC 3.2.1.30).

\section{MATERIEL ET METHODES}

L'étude a été effectuée sur des éjaculats provenant de consultants pour infécondité du couple. Les dosages ont été réalisés sur le plasma séminal de :

- 18 sujets au spermogramme normal

- 32 sujets azoospermiques dont 1 cas d'agénésie déférentielle bilatérale.

Par ailleurs, 12 éjaculats fractionnés ont été analysés. Les activités enzymatiques ont été déterminées sur le plasma séminal obtenu par centrifugation de l'éjaculat, par des méthodes fluorimétriques précédemment décrites (7) et ont été exprimées en unités (une unité correspondant à la libération d'une nanomole de méthylumbelliférone par minute et par ml. De plus, les profils enzymatiques ont été déterminés par isoélectrofocalisation sur plaques (Ampholines PAG plaques, Pharmacia-LKB) (8). Pour les éjaculats fractionnés, les concentrations en zinc déterminées par absorption atomique sont exprimées en $\mu$ mol.l-1.

\section{RESULTATS}

Un travail préliminaire avait permis d'établir les valeurs usuelles de la $\beta$-MAN, $\alpha$-FUC et NAG dans le plasma séminal à partir de 18 éjaculats considérés comme normaux (7). Par ailleurs, pour les éjaculats fractionnés, les valeurs des activités enzymatiques ont été confrontées et comparées au taux de zinc choisi comme marqueur prostatique (Tableau I). Actuellement, notre travail concerne l'étude de ces glycosidases dans des cas d'azoospermie. Une première analyse a porté sur une série de 32 sujets azoospermiques (Tableau II), la cause de l'azoospermie étant obstructive pour certains d'entre eux. Parallèlement, un cas d'agénésie déférentielle bilatérale a été étudié (Tableau III).

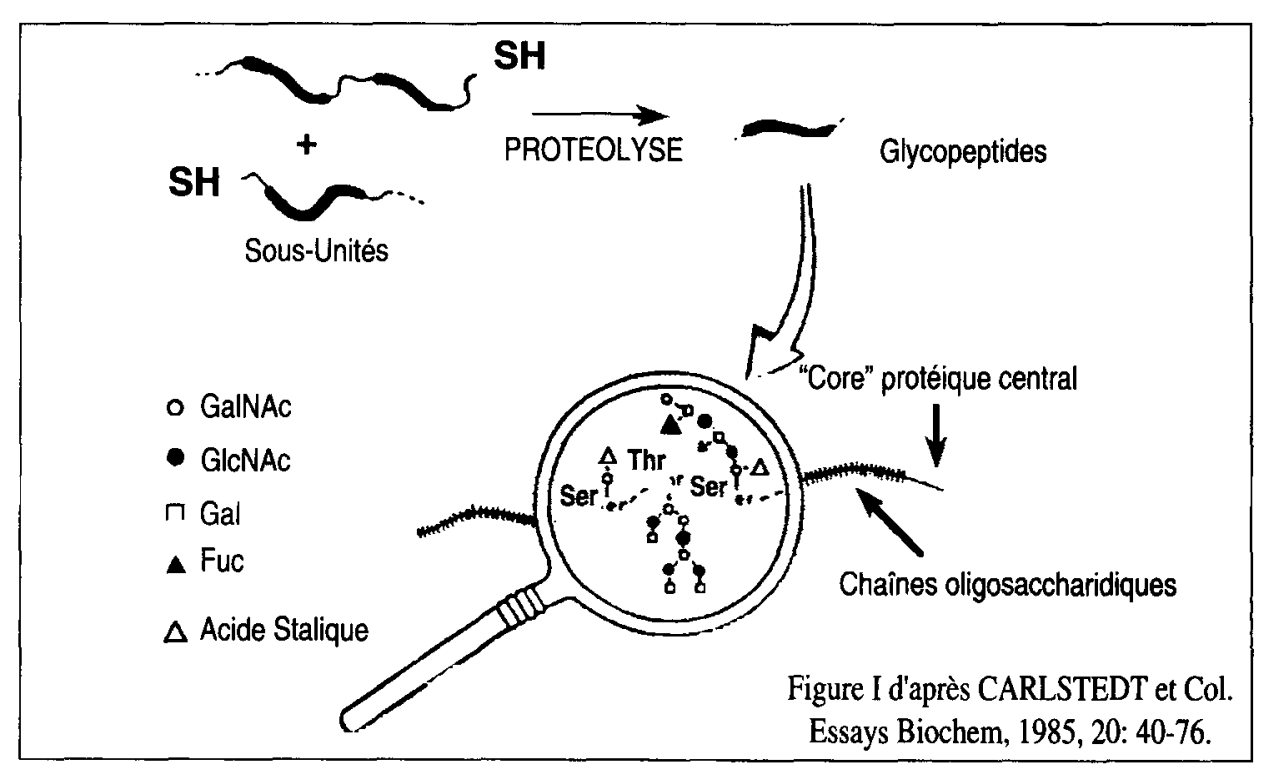


Tableau I : Activités des glycosidases dans le plasma

séminal normal et dans des éjaculats fractionnés

\begin{tabular}{|c|c|c|c|c|}
\hline $\mathrm{n}=18$ & \multicolumn{2}{|c|}{$\beta$-D-MAN } & $\alpha-L-F U C$ & NAG \\
\hline unités & \multicolumn{2}{|c|}{$70 \pm 23$} & $151 \pm 70$ & $1432 \pm 481$ \\
\hline \multicolumn{5}{|c|}{ unité = une nmole de méthyl-umbelliférone libérée par mn et ml). } \\
\hline & $\beta$-D-MAN & $\alpha-L \cdot F U C$ & NAG & $\operatorname{Zinc}(\mu \mathrm{mol} / \mathrm{l})$ \\
\hline Fraction 1 & $72,8 \pm 24,3$ & $241,6 \pm 53,1$ & $1337,1 \pm 370,4$ & $3566 \pm 1273$ \\
\hline Fraction 2 & $55,9 \pm 28,4$ & $186,9 \pm 41,7$ & $761,5 \pm 251,6$ & $2516 \pm 1322$ \\
\hline $\mathrm{p}$ & NS & $0,05<p<0,01$ & 0,01 & 0,01 \\
\hline
\end{tabular}

Tableau II : Activités des glycosidases (en unités)

dans le plasma séminal normal et en cas d'azoospermie

\begin{tabular}{lcccc|} 
& & $\beta$-D-MAN & $\alpha$-L-FUC & NAG \\
Normal & $\mathrm{n}=18$ & $70 \pm 23$ & $151 \pm 70$ & $1432 \pm 481$ \\
Azoospermie & $\mathrm{n}=31$ & $36 \pm 17$ & $148 \pm 127$ & $811 \pm 583$ \\
\hline
\end{tabular}

Tableau III : Un cas d'agénésie déférentielle bilatérale

\begin{tabular}{|ccc|}
\hline \multicolumn{3}{c|}{ Fructose : absence } \\
& Zinc, phosphatases acides : normaux & \\
& Carnitine $: 25$ nmoles $/ \mathrm{L}$ & \\
$\beta$ B-D-MAN & $\alpha-\mathrm{L}-\mathrm{FUC}$ & $\mathrm{NAG}$ \\
15 unités & 126 unités & 756 unités \\
\hline
\end{tabular}

\section{DISCUSSION}

Les glycosidases qui ont fait l'objet de notre étude sur le plasma séminal sont lysosomiques, c'est à dire qu'elles sont présentes aussi dans l'acrosome des spermatozoildes dont on sait l'origine golgienne. Pourtant une activité enzymatique est mise en évidence dans le plasma séminal de sujets azoospermiques. L'analyse d'éjaculats provenant d'azoospermie excrétoire serait particulièrement intéressante si une exploration chirurgicale a été faite. Nous n'avons pas encore réalisé, pour des raisons techniques, l'analyse d'éjaculats de sujets vasectomisés. Ce travail est en cours mais quelques faits semblent déjà se dégager :

- Le taux de NAG est significativement plus élevé dans la première fraction que dans la seconde $(\mathrm{p}=$ $0,01)$. Il en est de même pour le zinc qui est un marqueur prostatique. Il semble donc possible de considérer la NAG comme un marqueur des sécrétions prostatiques.

- L'activité $\beta$-MAN est significativement plus basse en cas d'azoospermie quelle qu'en soit l'origine. Une explication doit être recherchée. Il n'y a pas de corrélation entre l'activité de cette enzyme et le nombre de spermatozoïdes par éjaculat.

- Le taux d' $\alpha$-FUC n'est pas modifié dans le

\section{REFERENCES}

1 - Gonzalès J. Aspects biorhéologiques et biochimiques de la liquéfaction de l'éjaculat humain. Bulletin de la Société d'Andrologie de Langue Française, 1990, 4 : 49.

2 - Conchie J., Mann.T. Glycosidases in mammalian sperm and seminal plasma. Nature, 1957, $179: 1190$.

3 - Tremblay R.R., Chapdelaine P., Mailhot J. $\alpha 1-4$ glucosidase activity in human semen : variations with number and mobility of spermatozoa. Fertil. Steril., 1979, $31: 592-593$.

4 - Guerin J.F, Ben Ali H., Rollet J., Souchier C., Czyba J.C. - $\alpha$-Glucosidase as a specific epididymal enzyme marker : its validity for the etiologic diagnosis of azoospermia. J. Androl., 1986, $7:$ 156-162.

5 - Viljoen M.H., Bornman M.S., Van Dermerwe M.P. and Du Plessis D.J. Alpha-glucosidase activity and sperm mobility. Andrologia, 1990, 22 : 205-208.

6 - Cooper T.G., Yeung C.H., Nashan D., Jockenhovel F., Nieschlag E. Improvement in the assessment of human epididymal function by the use of inhibitors in the assay of $\alpha$-glucosidase in seminal plasma. Int. J. Andrology, 1990, $13: 297-305$.

7 - Ben Ayed S., Gonzalès J., Foglietti M.J., Percheron F. and Bernard M. On some glycosidases $(\beta-D$ mannosidase, $\alpha$-L-fucosidase, $\mathrm{N}$-acetyl- $\beta$-D-glucosaminidase) of human seminal plasma. Andrologia, 1989 , $21: 432-436$

8 - Colin B., Bernard M., Fogletti M.J., Percheron F. BD-mannosidase in human polymorphonuclear leucocytes and lymphocytes : a comparative study. Int. J. Biochem., 1987, $19:$ 395-397.

RESUME : La liquéfaction du sperme correspond à une protéolyse touchant notamment des glycoprotéines et pourrait également s'accompagner d'une lyse au niveau des branchements glycaniques impliquant l'action de glycosidases. Parmi elles, l' $\alpha-1-4$ glucosidase a été plus particulièrement étudiée. Elle constitue un bon marqueur épididymaire, mais son rôle dans la liquéfaction n'est pas établie. Le but de ce travail est de doser d'autres glycosidases séminales : la $\beta$-D-mannosidase, l' $\alpha$-L-fucosidase et la $\mathrm{N}$-acétyl- $\beta$-D-glucosaminidase. Ces glycosidases provenant du tractus génital, nous avons cherché si elles pouvaient servir de marqueurs.Il ne semble pas exister de corrélation entre les activités de ces trois enzymes et le nombre de spermatozoïdes. Même en cas d'azoospermie, les activités enzymatiques sont toujours mises en évidence. Une série de 32 éjaculats provenant de sujets dont l'azoospermie relevait d'étiologie secrétoire ou excrétoire a fait l'objet de cette analyse. Les différences observées portent à la fois sur l'activité enzymatique totale (l'activité $\beta$-MAN est significativement diminuée) et sur les proportions relatives des différentes formes enzymatiques, et ce, en fonction de l'étiologie de l'azoospermie. MOTS-CLES : Plasma séminal humain - Glycosidases - $\alpha$-L-fucosidase - $\beta$-D-mannosidase $-\mathrm{N}$-acétyl - $\beta$-D-glucosaminidase - Azoospermie.

ANDROLOGIE, 1991, 1 :13-14 